\title{
How can a box become a garment
}

This paper analyses the pedagogy connecting fashion students' understanding of pattern making and garment construction through the project 'How can a box become a garment', implemented as an alternative novel approach for students new to pattern construction in how to transform 2D material into a 3D garment, creating unconventional shape and form.

Using flattened boxes as a substitute for pattern pieces and working in pairs, students were briefed that there was no right or wrong response, but rather to focus on creating interesting 3D form. Working with a minimum of two boxes cut out in calico, students were encouraged to progress organically alternating between stitching random edges together and analysing the resulting shape on a mannequin until they deemed their garment complete.

The research gathered highlights how alternative teaching approaches to creative pattern cutting can be complementary to traditional skills, in particular to students who find this area challenging.

Keywords: Creative pattern cutting, pedagogy, 2D-3D, fashion design.

\section{Introduction}

Pattern cutting and garment manufacture are the processes in fashion whereby the designed garment is translated. A specification drawing is interpreted (Campbell, 2014) into a set of flat paper or cardboard pattern pieces that, when cut out in fabric and sequentially stitched together, creates the garment.

For fashion students who have never constructed a garment before, the whole process can be overwhelming, having to master the skills of pattern cutting, lay planning, cutting out, sewing, overlocking and pressing, and furthermore comprehend the new terminology of notches, grainlines, scye, toiles, facings, and so on; negative first impressions may be formed that can bring about aversion to the topic.

To the novice, realising his/her garment designs can be a challenge. Pattern 
cutting alone, not only deals with the complexity of working two dimensionally whilst thinking and visualising in 3D (Aldrich, 1997), but also one must consider the size and complex shape of the human body and its relationship with the garment allowing for ease and fit. The cut, silhouette, proportion and design features of the garment require precise interpretation to ensure that the essence of the design is not lost in translation. Understanding needs to be given to fabric characteristics, the drape, hang and movement required of the final garment, pattern pieces have to be drafted in such a way they correspond when stitched together (Lo, 2011) and all this whilst ensuring a high level of precision. Competency in pattern cutting takes a lot of intuition that can only come through understanding the surrounding influences that affect the final pattern.

Constructing their designs can be a demanding time for students; referring to pattern cutting, Parish (2013) states "frustration often arises when understanding and skills are insufficient for translating the concept into a physical item" (p. 8), and Ashdown (2013) writes "when a student faces a sewing machine or a muslin [toile] and a dress form for the first time...it can be a difficult task to convince them that they can and should work to gain these skills" (p.116). Numerous sample toiles are often required to realise the design as it was envisaged and although this can frustrate students, this perseverance brings with it experience and practice.

Engagement is paramount to master these skills as for most, competence and technical ability takes time, effort and practice. However, many talented fashion students may fail to reach their full potential as they cannot grasp the technical skills required to realise their designs; various reasons can affect this, for example, the complexity of transforming $2 \mathrm{D}$ to $3 \mathrm{D}$, the inability to operate in the linear manner required for the tasks or dyslexia, dyscalculia or, dyspraxia. Smith (2013) comments "many talented design students come out in a cold sweat at the sight of a pattern 
master" (a curved ruler used for pattern cutting and grading). As a result, designs can be diluted during either the development process or interpretation from illustration to garment, potentially losing proportion, scale, cut, silhouette or detailing, resulting in the desired aesthetic not being achieved.

Until recently, literature instructing how to pattern cut was limited, with many students adopting Winifred Aldrich's, Metric Pattern Cutting for Womenswear as their main reference. This book, when first published in 1976, entered the market when fashion in terms of industry, education and style was very different from what it is today. To a current fashion design student who is being asked for innovation, something that has not existed before, what they design will often not exist in any of these books. However, these books are still to be valued as an important learning tool to a pattern cutter, provided the user understands that it is up to them to identify and translate the pattern cutting techniques into their individual designs (Almond, 2011). Recently, the variety of books on the topic has increased; new literature on the subject is aligned more with contemporary fashion, giving less importance to pre-determined styles and more emphasis on the techniques used in pattern cutting (Lindqvist, 2014).

Fashion, and its incessant need for innovation (Breward, 2003), sees garments being pushed to the limit in terms of creativity and with this comes the need for highly sophisticated levels of traditional and innovative pattern cutting and sewing skills. However, it is impossible to teach students all there is to know about pattern cutting and garment manufacture when not all garments have been conceived yet. Almond (2010) supports this when he states "it should be emphasised, however, that pattern cutting is never completely mastered" (p.17). Designing garments that have not existed before brings new technical challenges to the maker that need to be resolved through past experience, problem-solving and trial and error. As fashion drives creativity in the 
designer, that of the pattern cutter follows suit and a skill that was originally referred to as a craft (Aldrich, 1997) has become recognised more recently as an art (Lo, 2011 and Parish, 2013).

In recent years, new techniques and methods have been introduced to the platform of traditional pattern cutting to provide alternative methods of creating innovative garments, for example, Julian Robert's subtraction cutting, zero waste pattern cutting or Shingo Sato's transformational reconstruction. For students who struggle with traditional pattern cutting, utilising an alternative method or creating their own system can be beneficial to realise their full potential. Almond (2011) states "students need to explore as many pattern cutting techniques as possible to achieve their shapes" (p.67).

Educating students in technical skills, it is important to direct students in becoming independent thinkers, to enable their lifelong learning. Once graduated, technical support ceases and literature will rarely provide the exact instructions required to create their designs. As discussed above, fashion design is a topic that is continually evolving, producing garment designs that have not existed before and as such, the need for independent thinking, lifelong learning and problem-solving skills are paramount to achieve the desired outcome.

\section{Background}

To strengthen connections between drawing and studio practice, the 'How can a box become a garment' project was developed. The pedagogical underpinning of the overall project was to expose the connection between drawing for idea generation, the making process and the final presentation stages within the design process, using the box as a common denominator throughout. The author will focus this paper primarily upon the workshop covering the making element of this project; however, drawing sessions will 
also be included where applicable to aid communication of the project.

Through experience, the author has noticed that many students in the early stages of their education pattern cut and stitch their way through a project using any combination of books, online tutorials or technical support to create their designs without really understanding the processes they are undertaking. It is not until manufacture is complete and students can see for themselves the finished garment that they then comprehend why they have carried out the production processes.

New students participate in intensive technical skills workshops; however, resource constraints do not allow for traditional pattern cutting to be taught in a manner that correlates with the corresponding sewing technique. When these two disciplines are brought together for the first time, students are usually faced with constructing their designed garment and although they navigate their way through the stages of construction, it often takes reflection upon the finished garment to appreciate the processes involved in creating their garment. Conscious of the complexities faced when learning to pattern cut and manufacture garments, and seeking to introduce students to experimental as well as traditional techniques, the 'How can a box become a garment' project was the ideal platform in which to introduce fashion technical skills to new students.

Despite the increasing popularity of craft pursuits, for example, dressmaking, knitting and quilting, over the last five years (Hall and Jayne, 2014), the demise of these skills in the years preceding this resurgence has resulted in many millennial generation students enrolling on a fashion course with no past experience of home craft making or having made a garment. Creating what for many is their first garment, the three-hour workshop was developed to enable students to quickly understand the construction process right at the outset of their technical skills education. The design system "a 
mixture of curiosity, construction, sharing and playing" (Von Busch, 2008, p. 36) was applied and a simplified manufacture methodology adopted to provide an enhanced learning experience in a non-judgemental environment.

There are many theories associated with how students learn. This project has at its foundation the ripples in the pond theory by Race (2005) where the stages of learning follows wanting, needing, doing, feedback, making sense, coaching and assessing at each stage of the learning process. This theory acknowledges a greater level of flexibility towards how each stage of learning not only influences the next but can also interact with its predecessor and successor to complete the learning experience.

A deep approach to learning is encouraged by linking new knowledge to previous knowledge throughout the project and promoting engagement in the project tasks with all students actively being involved in the learning activities. This creates a positive and enjoyable learning environment where teaching encourages interaction between tutor and students through the discussion of work and the provision of feedback as the project progresses during the three sessions. In this way, a studentcentred model of teaching was adopted with the teaching supporting the learning (Biggs and Tang, 2007).

The 'How can a box become a garment' project is underpinned with this learning theory and learning style. The pedagogy at its foundation believes that creating sculptural garments incorporating shape and volume does not always have to be a complex process. The project aims to act as an icebreaker to technical skills, utilising improvised pattern pieces, draping and unplanned sewing, to introduce students to the manufacture process whilst minimising its complexities. Campbell (2014) suggests "creating volume using an experimental approach to design through pattern cutting could help students gain a better understanding of garment shape making, and thus 
become a more accomplished fashion designer" (p.4), supporting the pedagogical theory of the project.

Condensing the process of manufacturing a garment into a three-hour session, the project aims to be a catalyst to the finished garment point, providing the "eureka moment' at the start of students' technical skills education. The project was designed to improve engagement throughout garment production in subsequent projects, through quickly providing students with an outcome that could be reflected upon. This is to provide an understanding of the production processes required to construct a garment. Students appreciate that the 'doing' element of the learning cycle precedes the 'making sense' component (Race 2005) through experience gained by participating in this project.

\section{Methodology}

The 'How can a box become a garment' project consists of three separate taught sessions; firstly, students participate in a silhouette analysis drawing class followed by a manufacture workshop where they create a sculptural garment and conclude the project with a fashion illustration drawing class.

'How can a box become a garment' was delivered during academic session 2014/2015 to year two BA (Hons) fashion and textiles students at Gray's School of Art, Robert Gordon University. First year is a foundation year, and as such, no exposure to pattern construction and garment manufacture was included within the curriculum. The cohort contained a mixed range of pattern cutting and garment manufacture experience: most students were novices with no previous exposure to these areas, though a minority had technical skills gained whilst enrolled on previous college courses. 


\section{Drawing: silhouette analysis}

Using a clothed life model positioned in the middle of the drawing studio, students were tasked with creating an interesting silhouette around a $360^{\circ}$ rotation by draping the human body with a combination of made up and flattened out boxes (see Figure 1). The three-hour drawing session focused on analysis of the relationship between the body and the materials draped around it. Emphasis was given to how garments commence and conclude with the human body (Lindqvist, 2014), and visualising the silhouette to consider the possibilities of how the boxes could become a garment. Students worked in a medium of their choice to create interpretative drawings (see Figure 2). During the drawing session, the pose of the model and the configuration of the boxes changed four times.

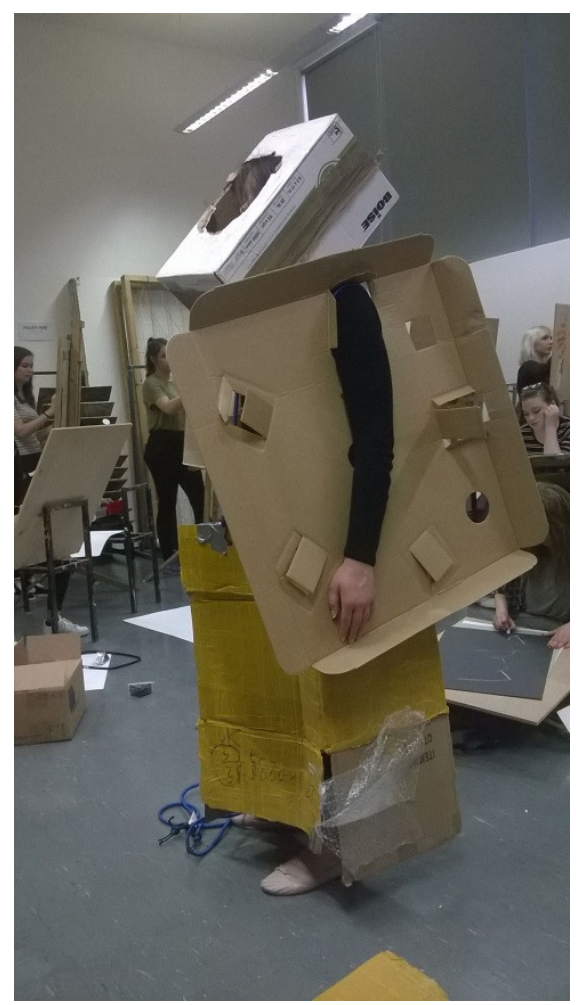

Figure 1. Drawing: silhouette analysis, interaction between the body and boxes photograph. (C) [Katie Morgan] reproduced by permission of Katie Morgan.

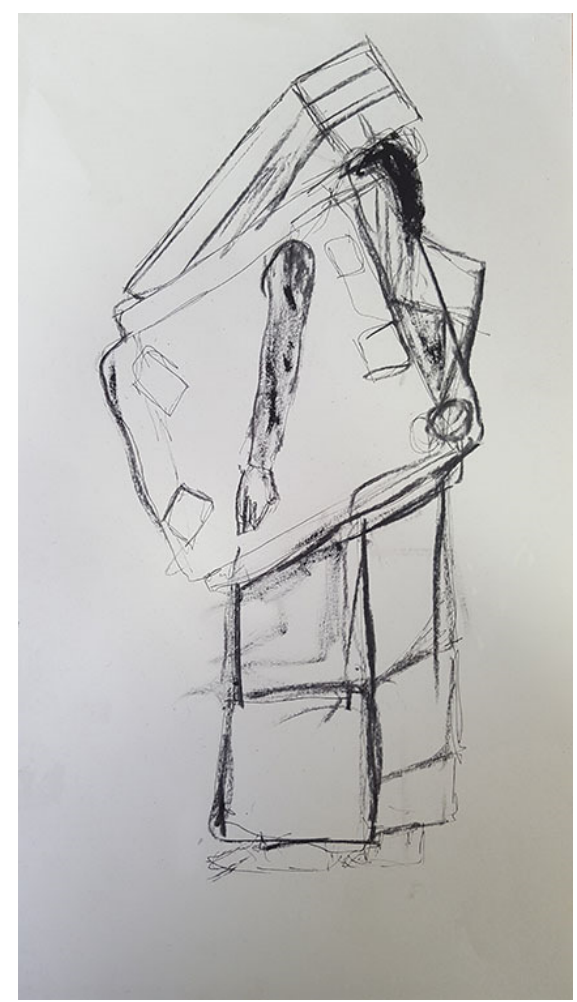

Figure 2. Drawing: silhouette analysis, interaction between the body and boxes drawing. (C) [Tilly Langworth] Reproduced by permission of Tilly Langworth 


\section{Manufacture workshop: sculptural garment}

All boxes used in the drawing session were pre-flattened in preparation for the workshop, calico was cut to lengths of three metres for each garment and mannequins were readily available. The three-hour workshop took place in the fashion manufacture room.

Through discussion, a set of step-by-step images and a sample garment, students were briefed on the process they were to follow, the learning outcomes and expected resolution of a sculptural garment. The garment did not have to conform to the conventional expectation of what a garment is and could take any form. Students were advised to be inspired from their preceding drawing session relating to silhouette analysis and the $360^{\circ}$ rotation of the body. Questions were welcomed; however, none was asked and students were happy to proceed with the instructions provided.

Working in self-selected groups of two to support peer-to-peer learning, students were advised to select no less than two flattened out box templates. Students lay planned these onto calico and drew around the templates following the contour to include all details, before cutting these

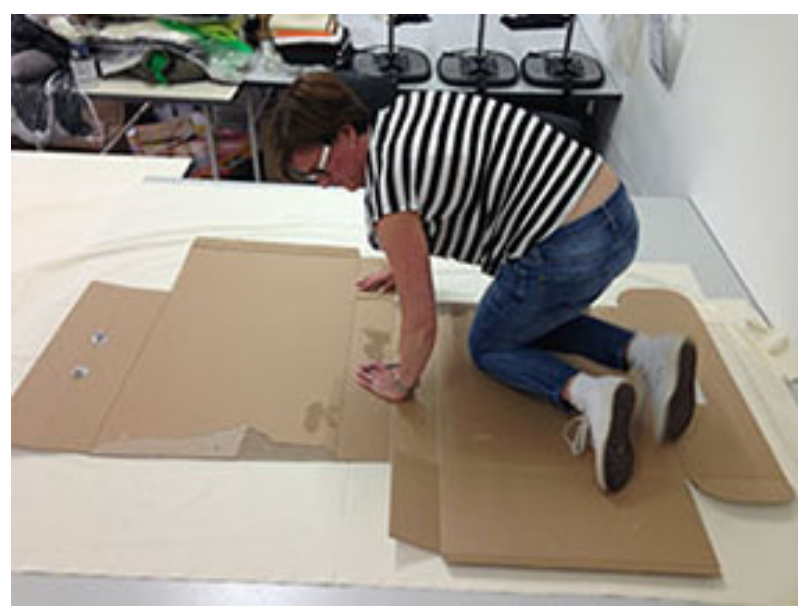

Figure 3. Lay planning box pattern pieces. () [Charlie Hackett] Reproduced by permission of Charlie Hackett.

out (see Figure 3). The flattened out boxes provided an alternative means of creating pattern pieces for garments without the prerequisite to pattern cut.

During the briefing, students were advised not to remove any calico from their cut out box patterns; the only removal of cloth permitted was apertures for the neck, arms or legs and although these openings were required, these did not have to be in the 
classic sense. Modesty was not an issue and if an area on the mannequin was not covered when normally you would expect it, then this was fine. Quality also was not required to be at the high standard normally expected of students with innovation and creativity being far more important for this project.

The main aim of the workshop was to create interesting three-dimensional sculptural shapes, working spatially around the body of the mannequin and not to the figure. It was stressed to students that there were no right or wrong answers to the workshop, not to over-think their next move, but to be creative, organic, innovative, random, spontaneous and most of all to have fun and enjoy the process.

Once all their box pattern pieces were cut out (see Figure 4), the next stage was to take the individual pieces and stitch them together along one edge in any way they wanted, to create one piece of fabric. Mannequins were introduced to the process at this point and the students began creating their garment by draping the calico over the

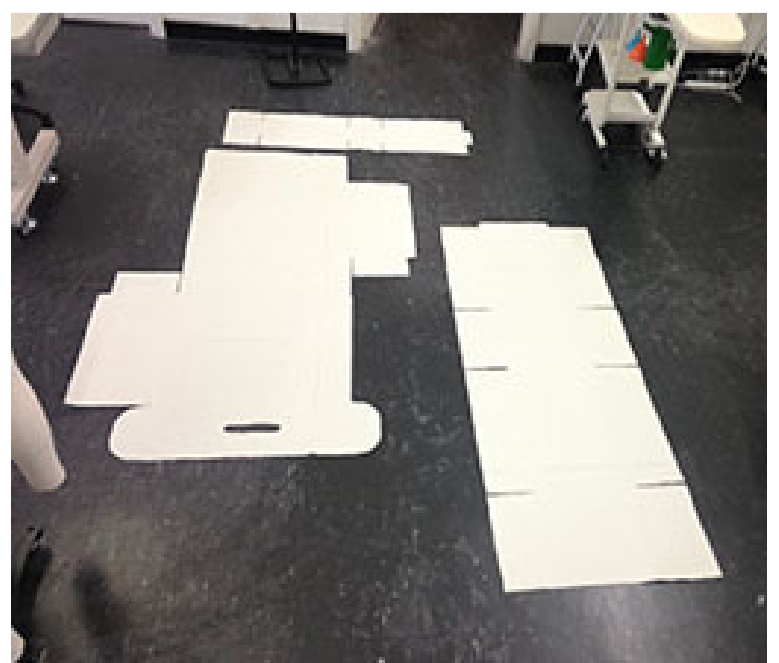

Figure 4. Cut out box pattern pieces. ( $)$ [Charlie Hackett] Reproduced by permission of Charlie Hackett

mannequins, selecting random pieces to join together, analysing the resulting shape before either re-selecting pieces to join or stitching them together (see Figure 5).

Progression of the garments continued by alternating between stitching areas together and evaluating the resulting shape on the mannequin until students considered their garments complete. Manufacturing a garment using this method provides an alternative to the pre-planned sequencing and operational breakdowns usually required. 
The workshop concluded with the mannequins dressed in the completed garments and lined up against a wall. Students were requested to step back and view their results, digest what they had achieved in a short period of time and analyse the sculptural shapes created and their relationship with the body. Tutors discussed how the garments created could be re-positioned on the mannequins, either back to front or upside down to analyse the aesthetics, scale and proportion of the sculptural shapes when viewed differently on the body.

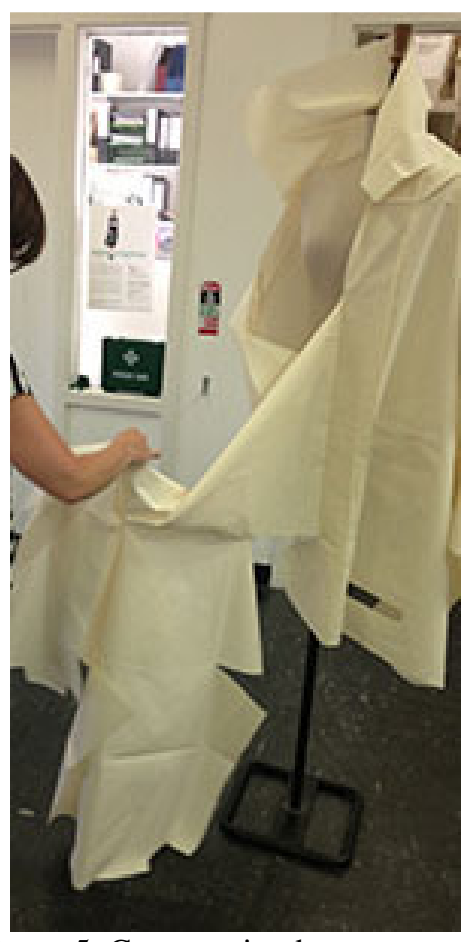

Figure 5. Construction box garment. (C) [Charlie Hackett] Reproduced by permission of Charlie Hackett.

\section{Drawing: fashion illustration}

A selection of the garments created in the manufacture workshop was utilised for the final session of the project. The garments were displayed on mannequins and positioned around the drawing studio (see Figure 6). Placement of the mannequins was carried out by students, with emphasis on the perspective being around the $360^{\circ}$ rotation of the garment, appreciating the torso of the mannequin as a cylindrical shape and not simply a front and back. The drawing session focused on the garments' silhouette, shape analysis and material interpretation, creating illustrative drawings working in pencil. During the drawing session, the positioning and rotation of the mannequins changed four times. 


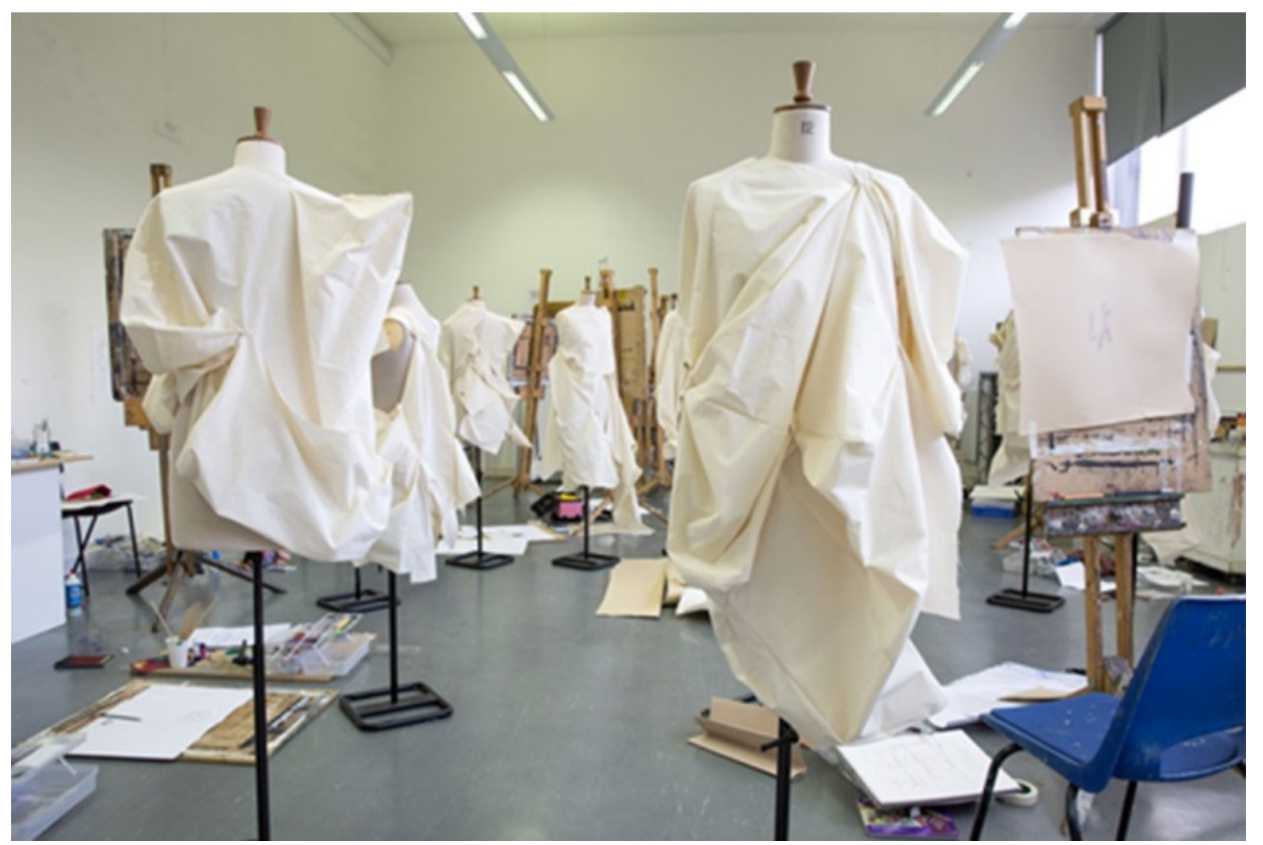

Figure 6. Drawing: fashion illustration, garments positioned for drawing. (C) [Fiona Stephen] Reproduced by permission of Fiona Stephen.

\section{Student evaluation}

Kolb (1984) proposed that if we become better at using all stages of the learning cycle, notably including reflecting on experience, we will become better lifelong learners. Reflective practice is a valuable methodology for personal learning and development, to learn from our past, assess where we are now and improve our present and future (Lawrence-Wilkes and Chapman 2014).

To encourage this vital component of the learning process, anonymous qualitative questionnaires were distributed to the 40 students who participated in the project, of which, 20 completed responses were received. The questionnaire was compiled of 10 questions; for the purpose of this paper, only questions pertinent to the manufacture workshop have been included:

1. Thinking about the unconventional garment that you created: how has this project provided you with an understanding of the body and its relationship with how a garment could be worn?

The replies predominantly refer to students' newfound appreciation of the 
possibilities of what a garment can be, with one student stating 'I was left with an understanding that garments don't have to be any particular thing' and another: I now understand that a neckline doesn't have to be a neckline, a armhole doesn't necessarily need to be at the side of the body and that the function of a garment does not need to be how we view them every day,

whilst a third wrote "the project showed me how different shapes can change the way a body can look dramatically'. Underlining that fashion does not always have to follow the context of being wearable, as exemplified in the work of Issey Miyake:

More than any other contemporary fashion designer, Miyake's work has become familiar through the process of reproduction, outside the traditional fashion system. Whether photographed as de-contextualized objects....or frozen as contemplative sculpture in one of many successive museum installations, Miyake's clothing designs have sought to transcend the stylistic, physical and temporal parameters of fashion per se, (Breward, 2003 p. 92).

2. In what ways did this project encourage you to be innovative in your approach to creating garments?

The majority of students commented that innovation was encouraged by the experimental, expressive and fun approach to the project; by working with the flattened out boxes, conventional shaping was not practical and therefore inspired creativity in their garments. One student states, 'it has encouraged me that there is no wrongs when it comes to designing and that perhaps the best outcomes actually come from experimenting or indeed by mistake'. Whilst another commented: focussing primarily on the silhouette is not an approach I have taken before. Thinking about the shapes the garment creates has expanded my design thinking 
and made me approach garments differently, as before I would focus on colour and pattern, and perhaps practicality. The designs I have created as a result of this approach are wildly different from anything I imagined at the beginning of the project.

3. Has this project impacted on your approach to fashion garment design and construction, please explain?

The responses all alluded to the project having a positive effect on the student's subsequent approach to fashion design and construction, opening up a new meaning to what a garment can be. Comments confirmed that for the majority, the project had furnished them with the confidence to leave behind the conventional and be far more innovative within their fashion design ideas, utilising their newfound appreciation for silhouette, three-dimensional shape and conceptual fashion. One student commented that they 'no longer look at fashion in a conventional way, but sees it more as shapes which can be moulded and moved' and another states, 'it has shown me that there is design potential in just about anything if you look at it the right way' whilst a third wrote, 'it has made me design my skirts more successfully, now that I can imagine them as garments in my head as I draw them'.

\section{Results and discussion}

The humble box was deemed most suitable for this project as the construction of both a box and a garment follows similar dimensional transformation, starting out as a twodimensional material (cardboard/pattern paper/fabric), transforming into a threedimensional structure (box/garment), before returning to a two-dimensional form (flattened boxes/folded clothes). As boxes are in plentiful supply, utilising this free item resulted in no cost being accrued from the course budget and old boxes were easily sourced. The material characteristics of a cardboard box were thought to be the most 
appropriate material for silhouette analysis drawing due to its rigid structure. Boxes come in many sizes and as such, the proportion and scale relating to the size of the box can be selected to align with that of the human body.

The template shape of the flattened out boxes works well as a substitute to pattern pieces, as incorporating the outline, flaps and cut out handles give numerous options as to what parts to stitch together to provide volume, shape and form. Modelling on the stand with a class of students using a straight length of fabric will usually see a few falling into the cliché of creating a toga or forgetting to incorporate a back, sides or both. The preceding silhouette analysis drawing class, working with the $360^{\circ}$ rotation of the body and using the box templates for pattern pieces, eliminated the predictable pitfalls that often result when students are faced with a length of cloth and a mannequin.

Using boxes as pattern pieces highlighted to students that there is design potential in just about anything. Creating sculptural garments from an item that most would deem finished with rubbish demonstrates the design value that can exist in everyday items.

Although a sample garment was available for students to view in relation to the required outcome, a small number of students began creating their garments using the form of the mannequin to shape the garment. Supervision provided the opportunity to discuss individually with each group the importance of including volume between the mannequin and the garment to achieve the project outcome of a sculptural garment focussing on silhouette. The garments created from medium and larger sized boxes were more successful in terms of creating sculptural silhouette, volume, shape and form in proportion to the body. The outcomes using smaller boxes lost volume due to their 
smaller size and groups working with these tended to have more detailing in their outcomes.

The experimental nature of this project did not suit all students: some found it difficult to be spontaneous and instead overly controlled the design of their garments. Working in groups was intended to alleviate this; however, where this difficulty was witnessed, tutors discussed with the group the benefits to being experimental and that by allowing the outcome to be dictated somewhat by chance, how unexpected shapes can be achieved.

The unorthodox method of garment manufacture here is in no way seen to be a replacement for traditional flat pattern cutting and garment manufacture. After all, the organic nature of this project whereby the outcome is somewhat down to chance simply would not work with a fully researched, developed and designed garment. This project is seen as introducing complementary skills to the traditional methods of manufacture, providing an understanding of what is possible when transforming $2 \mathrm{D}$ into $3 \mathrm{D}$ and as a method of familiarising the shaping required when pattern cutting to produce silhouette, volume, shape and form in garments.

The experience gained from completing the 'How can a box become a garment' project gave students greater confidence within their following design work. For many, the subsequent project was the first time they had taken their own design through to being a completed garment; under these circumstances, students can often hold back within their design ideas, fearing the unknown and the scale of the challenge they are setting themselves. However, designs for this project saw the influence of the boxes project with bold silhouette and shaping being tackled with little hesitance for the challenge ahead (see Figure 7). 


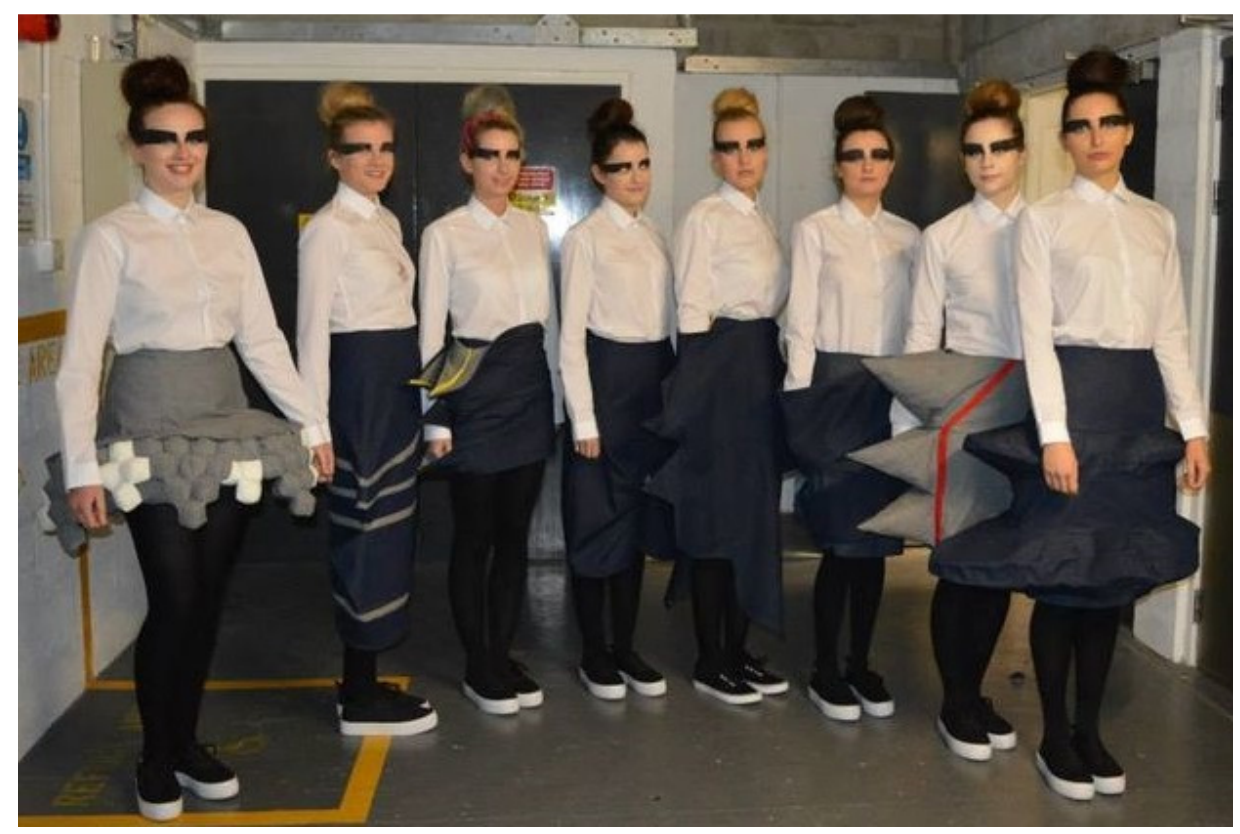

Figure 7. Denim skirt project. (C [Gabby O’Driscoll] Reproduced by permission of Gabby O’Driscoll.

Although not planned when the project was developed, additional learning outcomes were observed upon completion. A greater understanding of materiality was seen in subsequent projects, with higher levels of consideration to compatibility between design work and material characteristics. The overall formula of this project where unpacking, re-working and repackaging occurred embedded a problem-solving technique that some students applied to other areas of their learning. It was surprising to discover that many students had preconceived ideas of what a garment should be, thinking of garments in a more conventional sense; this project introduced a conceptual approach to what a garment could be, exposing students to the importance of experimentation and as such positively influenced future design work. A higher level of independent thinking and confidence relating to pattern cutting and garment manufacture was witnessed in students during succeeding projects in comparison to those in previous years who had not participated in the project.

The experimental approach this project entails provides a platform for students to recognise trial and error as an important factor in realising their designs. Error is not 
always a bad thing; 'happy accidents' often occur during the manufacture process when a student does not achieve the planned outcome but what has been created is preferred to the original: "Creativity comes from being bold, experimenting and allowing yourself to make mistakes" (Zaman, 2014, p. 8).

The continual critical analysis required to create the box garments highlights how the construction process is interlinked with design development and that this element of the design process does not necessarily cease in the layout pad. Evaluation from concept through to completion in design is a valuable asset and this project embeds this throughout all stages.

\section{Future development}

The knowledge gained by students during the 'How can a box become a garment' project could easily be transferred to other disciplines where understanding of two dimension, three dimension, spatial awareness, volume, silhouette, the human body, proportion, material characteristics, $360^{\circ}$, shape or form is required. The success of the project has led to the format being adopted by other design courses at Gray's School of Art. Further future development would see the 'How can a box become a garment' project introduced to other disciplines, for example, architecture, sculpture or engineering, either as an interdisciplinary or a subject-specific project.

\section{Conclusion}

Introducing a new skill that is complex and multi-layered can be overwhelming for students. It can often feel as though it takes an eternity to navigate through the production processes required to translate their designs into a completed garment, and negative first impressions may be formed that can bring about aversion to the topic. The author believes that by simplifying the process and introducing these skills in a fun, 
non-judgemental manner, students relate their first experience of constructing a garment as being an enjoyable and positive learning experience.

The project provides a shortcut to the 'eureka moment' where reflection on the completed garment provides students with a sense of achievement and an understanding of the manufacture processes undertaken. The experimental nature of this project, where the outcome was not what would be deemed conventional garments, eliminated the preconceived ideas of what a garment should be, leading to more innovation in future design work along with improved technical skills, material understanding, engagement, confidence and understanding of the human body.

Utilising the humble box as a concept to provide inspiration through drawing for idea generation, garment design and final presentation has exposed the design potential existing within everyday life and that the success or failure of a project can be determined by the journey the designer takes with his/her concept.

When technical skills do not come naturally, it is up to the individual to find or devise a system of creating garments that works for him/her. This project is highly beneficial in providing an alternative means of creating interesting shape, silhouette and form within a garment without ever having to pick up the dreaded pattern master.

\section{References}

Aldrich, W. (1997). Metric pattern cutting. Oxford: Blackwell Science Ltd.

Almond, K. (2010). Insufficient allure: the luxurious art and cost of creative pattern cutting. International Journal of Fashion Design, Technology and Education, 3(1), $15-$ 24. 
Almond, K. (2011). 'It looks very home dress-makey': strategies to include fashion students to realise a professionally finished garment: cut, construction, fabric, colour. Futurescan: Mapping the territory: Journal of Conference proceedings, 1, 64-71.

Ashdown, S. P. (2013). Not craft, not couture, not 'home sewing': teaching creative patternmaking to the iPod generation. International Journal of Fashion Design, Technology and Education, 6(2), 112-120.

Biggs, J. \& Tang, C. (2007). Teaching for quality learning at university. Maidenhead: Open University Press.

Breward, C. (2003). Fashion. Oxford:Oxford University Press.

Campbell, L. (2014). Volumetric shape making and pattern cutting. [Online] Retrieved from http://aut.researchgateway.ac.nz/handle/10292/8578

Hall, S. M., \& Jayne, M. (2014). Make, mend and befriend: geographies of austerity, crafting and friendship in contemporary cultures of dressmaking in the UK. Gender, Place and Culture A Journal of Feminist Geography, 1-19.

doi: 10.1080/0966369X.2015.1013452

Kolb, D. A. (1984). Experiential learning: experience as the source of learning and development. Englewood Cliffs, NJ: Prentice Hall PTR.

Lawrence-Wilkes, L. and Chapman, A. (2014). Reflective practice. [Online]. Retrieved from: http://www.businessballs.com/reflective-practice.htm

Lindqvist, R. (2014). The transformative cuts: new foundations in pattern cutting and approximations of the body. [Online] Retrieved from http://bada.hb.se/handle/2320/14454 
Lo, D. C. (2011). Pattern cutting. London: Laurence King Publishing Ltd.

Parish, P. (2013). Pattern cutting: the architecture of fashion. London: Fairchild Books.

Race, P. (2005). Ripples model: seven factors underpinning successful learning

[Online]. Retrieved from http://phil-race.co.uk/most-popular-downloads/

Smith, K. (2013, February). Pattern cutting for the petrified. Paper presented at The First International Symposium for Creative Cut, University of Huddersfield.

Von Busch, O. (2008). Fashion-able: hacktivism and engaged fashion design. Gothenburg: Johan Oberg.

Zaman, Z. (2014). Fashion pattern cutting: line, shape and volume. London: Bloomsbury Publishing Plc.

\section{Figures}

Figure 1. Drawing: Silhouette analysis, interaction between the body and boxes photograph. (C) [Katie Morgan] Reproduced by permission of Katie Morgan.

Figure 2. Drawing: Silhouette analysis, interaction between the body and boxes drawing. (C) [Tilly Langworth] Reproduced by permission of Tilly Langworth.

Figure 3. Lay planning box pattern pieces. (C) [Charlie Hackett] Reproduced by permission of Charlie Hackett.

Figure 4. Cut out box pattern pieces. (C) [Charlie Hackett] Reproduced by permission of Charlie Hackett.

Figure 5. Constructing box garment. (C) [Charlie Hackett] Reproduced by permission of Charlie Hackett. 
Figure 6. Drawing: Fashion illustration, garments positioned for drawing. () [Fiona Stephen] Reproduced by permission of Fiona Stephen.

Figure 7. Denim skirt project. (C) [Gabby O’Driscoll] Reproduced by permission of Gabby O'Driscoll. 